\title{
THYROID STIMULATING HORMONE AND THYROXINE SCREENING IN THE PRETERM NEWBORN:
}

\section{REFERENCE VALUES}

\section{Bagnoli Franco, Montecchiani Giada, Farmeschi Laura, Nappini Sara, Badii Silvia, Cecchi Sara, Mori Annalisa, Tomasini Barbara}

\author{
D.A.I. Maternal-Infantile, University of Siena, Siena, Italy
}

\begin{abstract}
The screening test for Congenital Hypothyroidism, performed in the 2nd - 3rd day of life, highlighted in the premature infant significant lower levels of thyroid stimulating hormone (TSH) and thyroxine (tT4) than those found in the full term newborn. Studies carried out after birth in the preterm infant, showed a persistence of low levels of thyroid hormones (TH) in the first two weeks of life, with a tendency toward spontaneous resolution at the end of the first month. This hormone deficiency has an incidence which is inversely related to gestational age (GA). However, there are no works which have evaluated the levels of TSH and tT4 in an appropriate large population of extremely low gestational age newborns (ELGANs) in the 2 nd - 3rd day of life divided by different GAs. To assess the levels of TSH and tT4 in the 2nd - 3rd day of life, from the lowest GA up to the end, in order to indicate the reference values which can be used to establish whether a replacement therapy with thyroid hormones in the premature infant is useful or not. In the present study we examined a total population of 1,671 adapted for gestational age newborns (AGA) of which 1,159 resulted in full term and 512 resulted in preterm. The population was divided into eight groups of GAs (23-25, 26-28, 29-30, 31-32, 33-34, 35-36, 37-38, 39-42 wks) and for each age group the median values of TSH and tT4 was determined. The TSH and tT4 were obtained in the $2 \mathrm{nd}-3 \mathrm{rd}$ day of life at the screening for Congenital Hypothyroidism. Looking at the whole population examined, the preterm infant presents significant lower levels of TSH $(p=0,00036)$ and tT4 $(p<0,00001)$ compared to the full term newborn in the 2 nd - 3rd day of life. TSH and tT4 levels increase gradually with the advancing of GA, reflecting the progressive maturation of the hypothalamicpituitary-thyroid axis. A linear and significant correlation $(p=0,001)$ was found between the levels of tT4 and GA. Instead, the TSH values which show an upward trend with the advancing of the GA, confirm a trend which is less clear. In preterm newborns, significant increases of tT4 levels are found for minimal increases of GA, suggesting that even in extremely premature infants (23-25 wks) there is already an appropriate function of hypothalamic-pituitary-thyroid axis, which matures rapidly and progressively. It is believed that even if the preterm newborn tT4 levels are lower than those found in a full term neonate, the metabolic needs of an ELGAN are sufficient. Our reference values in various GAs in preterm infants may be used to determine whether premature newborns may or not be required to make a substitution therapy, which in our opinion should be implemented when the tT4 levels are $<2$ SD for that gestational age.
\end{abstract}

Key words: screening test, TSH, tT4, full term and preterm newborn, reference values.

\section{INTRODUZIONE}

Lo Screening per l'Ipotiroidismo Congenito, introdotto negli anni '70, ha messo in evidenza livelli di tireotropina (TSH) e ormoni tiroidei (TH) differenti nei neonati prematuri rispetto ai nati a termine. Il neonato pretermine presenta una secrezione ormonale ridotta rispetto al neonato a termine, a causa della minor riserva tiroidea, della difficoltà ad alimentarsi, della ridotta sintesi epatica di TBG, dell'immaturità sia enzimatica che dell'asse ipotalamo-ipofisi-tiroide [1-3]. Il neonato prematuro di basso peso va incontro inoltre ad un'aumentata mortalità e morbidità perinatale, dovuta secondo alcuni autori (AA.) anche ai più bassi livelli di ormoni tiroidei, che persistono nelle prime 2-3 settimane di vita, con tendenza a risolversi spontaneamente entro il primo mese [3-8, 29,31]. Oltre a ciò, in questi neonati è riportato un peggior outcome neurologico a distanza [9]. Per tali motivi alcuni AA. hanno prospettato la necessità di somministrare ormoni tiroidei fin dai primi giorni di vita nel neonato di età gestazionale estremamente bassa (ELGAN)[10-20]. Tuttavia non sono presenti in letteratura lavori che abbiano valutato in $2^{\circ}-3^{\circ}$ giornata di vita, momento in cui i fenomeni di adattamento neonatale sono ormai completati, i livelli di TSH e tT4 in un numero sufficientemente ampio di soggetti ELGAN, suddivisi in varie fasce di età gestazionale (EG). I valori di riferimento ottenuti nelle varie fasce di età, possono essere utilizzati per stabilire l'opportunità o meno del trattamento ormonale sostitutivo del neonato estremamente prematuro.

Correspondence to:

Franco Bagnoli

Via Olanda 1 Carpineto, 53018 Siena, Italy.

Tel.: +39.0577.394169; Cell.: +39.349.088.6227; Fax: +39.0577.393547.

E-mail: bagnoli@unisi.it 


\section{SCOPO}

Stabilire i valori di riferimento del TSH e del tT4 in relazione all'età gestazionale.

\section{MATERIALI E METODI}

Nel presente studio abbiamo esaminato una popolazione totale di 1671 neonati AGA (adapted for gestational age) di EG media(wk) $=36,8 \pm 4,31$, PESO medio(gr) $=2936 \pm 983$, di cui 1159 nati a termine (EG media(wk) $=39 \pm 1,29$, Peso medio $(\mathrm{gr})=3587 \pm 315)$ e 512 prematuri $($ EG media $(w k)=31 \pm 3,12$, Peso medio(gr $)=1660 \pm 578$ ). Abbiamo escluso dal nostro studio i neonati le cui madri erano affette da patologie tiroidee e/o che fossero in terapia ormonale sostitutiva. La popolazione è stata suddivisa in 8 fasce di età gestazionale $(23-25,26-28,29-30,31-32$, $33-34,35-36,37-38,39-42$ wks), per ogni fascia di EG è stata calcolata la media \pm DS, la mediana ed i valori minimi e massimi del TSH e del tT4. Il TSH e il tT4 sono stati ottenuti in $2^{\circ}-3^{\circ}$ giornata di vita in occasione dello Screening per l'Ipotiroidismo Congenito, eseguito con sangue prelevato dal tallone ben riscaldato del neonato. Il tallone è stato spremuto delicatamente e la carta da filtro è stata appoggiata sulla goccia di sangue. In seguito i cartoncini sono stati lasciati asciugare all'aria per almeno 4 ore ed inviati al laboratorio entro 24 ore. La carta filtro utilizzata è stata prodotta da'Schleicher \& Shuell'.

Per il dosaggio ormonale è stato utilizzato il kit per il saggio fluoroimmunoenzimatico 'Delfia' (Pharmacia Turku Finland).

L'analisi statistica dei dati è stata effettuata utilizzando il sofware' Graph Pad Prism'.

\section{RISULTATI}

Nelle Tabelle 1-2-3-4-5-6 sono visualizzabili i livelli del TSH e del tT4 (media+- DS, mediana, valori minimi

Tabella 1.

\begin{tabular}{|c|c|c|c|c|c|}
\hline POPOLAZIONE & No. CASI & $\begin{array}{c}\text { EG (wk) } \\
\text { Media } \pm \text { DS }\end{array}$ & $\begin{array}{c}\text { PESO (gr) } \\
\text { Media } \pm \text { DS }\end{array}$ & $\begin{array}{c}\text { TSH } \\
(\mathrm{mUI} / \mathrm{L})\end{array}$ & $\begin{array}{c}\text { tT4 } \\
(\mu \mathrm{g} / \mathrm{dL})\end{array}$ \\
\hline $\begin{array}{c}\text { NEONATI } \\
\text { PRETERMINE }\end{array}$ & 512 & $31 \pm 3,12$ & $1660 \pm 578$ & $\mathbf{0 , 7 0 *}$ & $5,30^{* *}$ \\
\hline $\begin{array}{c}\text { NEONATI } \\
\text { A TERMINE }\end{array}$ & 1159 & $39 \pm 1,29$ & $3587 \pm 315$ & $1,00 *$ & $10,10 * *$ \\
\hline
\end{tabular}

No.casi., EG (media \pm DS), peso (media \pm DS), livelli di TSH e tT4 espressi come mediana nella popolazione esaminata di neonati a termine e pretermine. $\mathrm{TSH}^{*}(\mathrm{p}$ value $=0,00036), \mathrm{tT} 4^{* *}(\mathrm{p}$ value $<0,00001)$.

Tabella 2

\begin{tabular}{|c|c|c|c|c|c|c|c|c|c|}
\hline \multirow{2}{*}{$\begin{array}{c}\text { SUDDIVISIONE } \\
\text { PER } \\
\text { FASCE DI EG }\end{array}$} & \multirow[b]{2}{*}{$\begin{array}{l}\text { No. } \\
\text { CASI }\end{array}$} & \multicolumn{4}{|c|}{ TSH (mUI/L) } & \multicolumn{4}{|c|}{ tT4 $(\mu \mathrm{g} / \mathrm{dL})$} \\
\hline & & mediana & media $\pm \mathrm{DS}$ & v.min & v.max & mediana & media \pm DS & v.min & v.max \\
\hline $23-25^{\wedge} \mathrm{wk}$ & 37 & $\mathbf{0 , 5 8}$ & $0,69 \pm 0,63$ & 0,10 & 2,60 & 2,10 & $2,22 \pm 1,71$ & 0,00 & 6,60 \\
\hline $26-28^{\wedge} \mathrm{wk}$ & 91 & 0,70 & $1,18 \pm 1,36$ & $\overline{0,02}$ & 5,83 & 3,29 & $3,61 \pm 1,99$ & 0,20 & 8,60 \\
\hline $29-30^{\wedge} \mathrm{wk}$ & 84 & 0,94 & $1,42 \pm 1,84$ & 0,10 & 9,10 & 4,45 & $4,66 \pm 2,16$ & 0,60 & 10,60 \\
\hline $31-32^{\wedge} \mathrm{wk}$ & 99 & 0,80 & $1,20 \pm 1,18$ & 0,10 & 4,90 & 5,80 & $6,12 \pm 2,35$ & 1,80 & 19,40 \\
\hline $33-34^{\wedge} w k$ & 160 & 0,60 & $1,15 \pm 1,51$ & $\mathbf{0 , 0 0}$ & 8,80 & 6,70 & $7,10 \pm 2,74$ & 1,50 & 18,00 \\
\hline $35-36^{\wedge} \mathrm{wk}$ & 41 & $\overline{0,80}$ & $1,55 \pm 1,79$ & 0,10 & 8,00 & 6,70 & $\overline{7,09 \pm 2,38}$ & 3,60 & 12,90 \\
\hline $37-38^{\wedge} \mathrm{wk}$ & 613 & 0,90 & $1,43 \pm 1,72$ & $\overline{0,10}$ & 11,90 & 9,70 & $10,22 \pm 2,73$ & 4,30 & 22,10 \\
\hline $39-42^{\wedge} w k$ & 546 & 1,10 & $1,62 \pm 1,74$ & 0,10 & 10,70 & 10,40 & $10,65 \pm 2,48$ & 5,10 & 19,70 \\
\hline
\end{tabular}

Tabelle 2-3-4-5-6: Livelli di TSH e tT4 (mediana, media \pm DS, valori minimi e massimi) a varie fasce di EG. P value (TSH e tT4) in varie fasce di EG a confronto 
e massimi e $P$ value) in varie fasce di età gestazionale. Nelle Figure 1-2 è illustrato l'andamento dei livelli del TSH e del tT4, espressi come mediana, a partire dalle più basse età gestazionali fino al termine.

\section{DISCUSSIONE}

Nel presente studio abbiamo indagato la funzionalità tiroidea in un'ampia popolazione di neonati AGA, di EG compresa tra la $23^{\circ}-42^{\circ}$ settimana. Abbiamo preferito valutare i livelli di TSH e tT4 in $2^{\circ}-3^{\circ}$ giornata di vita, per limitare l'interferenza di fattori confondenti perinatali, quali il tipo di parto, l'ipossia o asfissia alla nascita e i fenomeni di adattamento neonatale $[21,22,28]$. Abbiamo così stabilito, in una popolazione sufficientemente ampia, i livelli di TSH e tT4 appropriati per le varie età gestazionali. In precedenza vari studi sono stati effettuati nel feto e nel neonato a termine e pretermine, per valutare l'influenza dell'età ge- stazionale sulla funzionalità tiroidea, sia prelevando il sangue fetale con funicolocentesi [23-25] o alla nascita con il prelievo di sangue cordonale [26-28].

Ballabio e coll. [23] hanno valutato i livelli di TSH e tT4 su sangue prelevato con funicolocentesi in 23 feti di EG compresa tra 18-31 wk e hanno evidenziato incrementi significativi dei livelli fetali di entrambi gli ormoni all'avanzare dell'EG. Analoghi risultati, su campioni di sangue ottenuti con funicolocentesi di 58 feti di EG compresa tra 17-37 wk, sono stati ottenuti da Thorpe-Beenston e coll. in uno studio del 1991 [24]. Nel 1998 Kilby e coll. hanno effettuato una ricerca su funicolocentesi di 11 feti di EG media 22 wk e 15 feti di EG media $31 \mathrm{wk}$ e hanno riscontrato come, tra il $2^{\circ}$ e il $3^{\circ}$ trimestre di gravidanza, vi fossero significativi incrementi del fT4 e fT3, ma più modesti e non significativi aumenti del TSH [25].

Alcuni studi hanno analizzato i livelli di TSH e tT4 alla nascita nel sangue cordonale [26-28].

Klein e coll. [26] hanno valutato una popolazione di
Tabella 3.

\begin{tabular}{|c|c|}
\hline EG & $\begin{array}{c}\text { P value }<\mathbf{0 , 0 5} \\
\text { TSH } \text { mUI/L }\end{array}$ \\
\hline 23-25 wk vs 26-28 wk & $\mathrm{P}=0,1053$ \\
\hline 26-28 wk vs 29-30 wk & $\mathrm{P}=0,1953$ \\
\hline 29-30 wk vs 31-32 wk & $\mathrm{P}=0,4563$ \\
\hline 31-32 wk vs 33-34 wk & $\mathrm{P}=0,0428$ \\
\hline 33-34 wk vs 35-36 wk & $\mathrm{P}=0,0268$ \\
\hline 35-36 wk vs 37-38 wk & $\mathrm{P}=0,3533$ \\
\hline 37-38 wk vs 39-42 wk & $\mathrm{P}=0,0085$ \\
\hline
\end{tabular}

Tabella 4.

\begin{tabular}{|c|c|}
\hline EG & $\begin{array}{c}\text { P value }<\mathbf{0 , 0 5} \\
\text { tT4 } \mu \text { g/dL }\end{array}$ \\
\hline 23-25 wk vs 26-28 wk & $\mathrm{P}=0,0001$ \\
\hline 26-28 wk vs 29-30 wk & $\mathrm{P}=0,0004$ \\
\hline 29-30 wk vs 31-32 wk & $\mathrm{P}=0,0001$ \\
\hline 31-32 wk vs 33-34 wk & $\mathrm{P}=0,0014$ \\
\hline 33-34 wk vs 35-36 wk & $\mathrm{P}=0,4676$ \\
\hline 35-36 wk vs 37-38 wk & $\mathrm{P}=0,0001$ \\
\hline 37-38 wk vs 39-42 wk & $\mathrm{P}=0,0002$ \\
\hline
\end{tabular}

Tabella 5.

\begin{tabular}{|c|c|}
\hline EG & $\begin{array}{c}\text { P value }<\mathbf{0 , 0 5} \\
\text { TSH } \mathbf{~ m U I / L}\end{array}$ \\
\hline 23-28 wk vs 29-36 wk & $\mathrm{P}=0,124$ \\
\hline 29-36 wk vs 37-42 wk & $\mathrm{P}=0,0099$ \\
\hline
\end{tabular}

Tabella 6.

\begin{tabular}{|c|c|}
\hline EG & $\begin{array}{c}\text { P value }<\mathbf{0 , 0 5} \\
\mathbf{t T 4} \boldsymbol{\mu} \mathbf{g} / \mathbf{d L}\end{array}$ \\
\hline 23-28 wk vs 29-36 wk & $\mathrm{P}<0,00001$ \\
\hline 29-36 wk vs 37-42 wk & $\mathrm{P}<0,00001$ \\
\hline
\end{tabular}

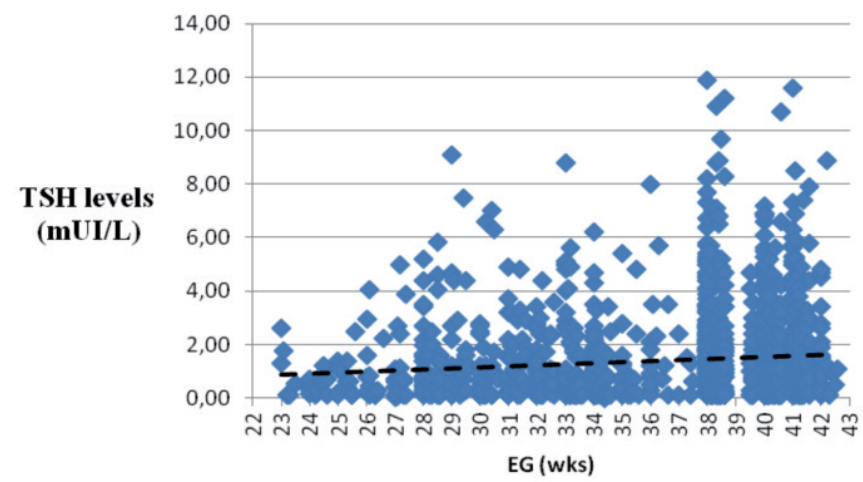

Figura 1.

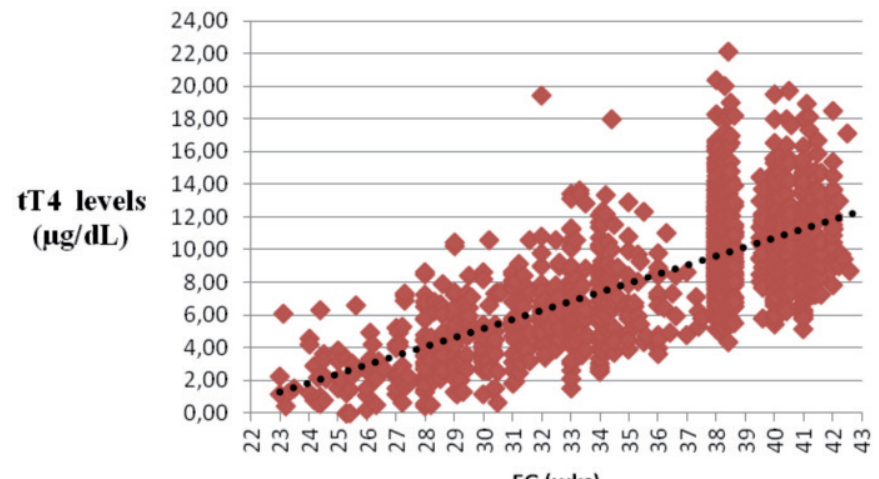

EG (wks)

Figura 2.

Figure 1-2: Andamento dei livelli di TSH e tT4, espressi come mediana, nei neonati di EG compresa tra $23-42 \mathrm{wk}$ in $2^{\circ}-3^{\circ}$ giornata di vita. 
neonati di EG compresa tra $26-43$ wk e hanno rilevato significativi incrementi di tT4, fT4 e TBG con l'avanzare dell'EG tra la $26^{\circ}$ e la $33^{\circ}-35^{\circ}$ wk $(p<0,001)$, ma non dopo la $34^{\circ} \mathrm{wk}$, quando tali parametri rimanevano stabili.

Bongers-Shokking e coll. [27] nel 1984 hanno esaminato i livelli di TSH e tT4 in varie epoche: alla nascita su sangue cordonale in 107 nati, a 24-30 h in 25 nati, a 6-9 giorni in 104 nati e a 13-20 giorni di vita in 57 neonati. Il tT4 mostrava una correlazione positiva con l'EG e con il peso sia alla nascita che nei periodi successivi; al contrario tali AA. non hanno riscontrato alcuna correlazione tra il TSH e l'EG o il peso alla nascita. Recentemente Rashmi e coll. [28] hanno analizzato in una popolazione di EG compresa tra la $28^{\circ}-42^{\circ} \mathrm{wk}$, suddivisa in tre fasce di EG (28-32wk)/(33-36wk)/(37-42wk), i livelli di TSH ottenuti dal cordone e hanno notato una correlazione negativa tra il TSH e l'EG.

Anche gli studi effettuati nei giorni successivi alla nascita suggeriscono una correlazione positiva tra l'EG e i livelli di tT4, ma risultati discordanti per quanto riguarda il TSH $[29,30,31]$.

Wassenaer e coll. [29] hanno eseguito uno studio durante le prime 8 settimane di vita su 100 neonati di EG $<30 \mathrm{wk}$. Hanno suddiviso la popolazione in 2 gruppi: $<28$ wk e $\geq 28$ wk e hanno dimostrato una riduzione simile dei livelli di tT4 con valori minimi registrati al $7^{\circ}$ giorno e incrementi simili fino al $28^{\circ}$ giorno in entrambi i gruppi, con livelli significativamente più elevati nei neonati di più alta EG. Il TSH invece decrementa in entrambi i gruppi nei primi 3 giorni, per poi aumentare dopo il $7^{\circ}$ giorno, in modo più evidente nei neonati di più bassa EG e infine stabilizzarsi al $28^{\circ}$ giorno di vita, tipico di un neonato sano. Biswas e coll. [30] hanno esaminato 128 neonati prematuri di EG $<30$ wk nelle prime 2 settimane di vita e hanno evidenziato livelli di TSH e tT4 inferiori rispetto ai valori rilevati nei neonati a termine, ottenuti da altri lavori. Suddividendo la popolazione in 3 diversi gruppi di EG: (23$25 w k)-(26-27 w k)-(28-29 w k)$, hanno riscontrato livelli di TSH e tT4 più elevati alle più alte EG. In un recente studio effettuato da Dilli e coll. [31] nel 2010, sono stati messi a confronto 2 gruppi di neonati di EG $<32 \mathrm{wk}$ : 26-29 wk vs 30-32 wk, durante le prime 4 settimane di vita. I livelli sierici di tT4, durante tutto il periodo di osservazione, sono risultati significativamente superiori nel gruppo di più alta $\mathrm{EG}$, mentre non sono state trovate differenze significative nei livelli di TSH.

Pertanto tutti gli AA. che hanno valutato i rapporti tra l'EG e i livelli di tT4, sono concordi nel ritenere che la funzionalità tiroidea del prematuro sia ridotta in maniera strettamente correlata all' EG, sia alla nascita che nei giorni successivi. Risultati non univoci sono stati riscontrati per quanto riguarda il TSH.

Gli studi effettuati per stabilire reference ranges per il neonato pretermine sono poco numerosi e la casistica esaminata alle più basse età gestazionali è in genere abbastanza limitata ed i valori di riferimento per il T4 ed il TSH sono stati valutati nel sangue del cordone ombelicale o successivamente al $2^{\circ}-3^{\circ}$ giorno di vita [32-35].

Adams e coll. [32] nel 1995 hanno esaminato 174 neonati di EG compresa tra 25-36 wk, di cui solo 45 $<30 \mathrm{wk}$ ed hanno analizzato i livelli di TSH e fT4 in tempi diversi durante la prima settimana di vita. Il loro studio ha evidenziato una correlazione positiva significativa tra fT4 ed l'EG e valori inferiori di TSH nel neonato pretermine rispetto al neonato a termine. Clark ha valutato i livelli di TSH e fT4 dopo la prima settimana di vita, esaminando solamente 14 bambini tra la $28^{\circ}$. $30^{\circ}$ wk di EG, mentre tutti gli altri avevano un'EG $>30$ wk. Lo stesso AA. riscontra nel pretermine livelli di fT4 simili a quelli di bambini o adulti [33].

Williams e coll. hanno effettuato vari studi nel neonato a termine e pretermine, sia su sangue cordonale che nel primo mese di vita. In un primo lavoro [34] hanno esaminato su sangue cordonale alla nascita i livelli di TSH e tT4 in 4 gruppi di età gestazionale diverse: 23-27 wk, 28-30 wk, 31-34 wk, $\geq 37 \mathrm{wk}$. , riscontrando livelli simili di TSH tra la $27^{\circ}-36^{\circ} \mathrm{wk}$; il tT4 mostra invece incrementi progressivi dalla $23^{\circ} \mathrm{Wk}$ fino alla $\geq 37^{\circ} \mathrm{wk}$.

In un ampio studio del 2005 [35] lo stesso AA., ha preso in considerazione una popolazione di 620 neonati di EG compresa tra 23-42 wk e ha valutato i livelli di TSH e T4 per ogni settimana di EG considerata. I risultati evidenziano bassi livelli di T4 alle più basse età gestazionali, con incremento a partire dalla $27^{\circ} \mathrm{wk}$ fino a termine, mentre i livelli di TSH sono risultati simili tra la $27^{\circ}-36^{\circ} \mathrm{wk}$ di EG, con un trend in riduzione nelle fasce di età estreme.

Da quanto sovra esposto, la letteratura fornisce dei reference ranges per tempi diversi rispetto a quelli da noi valutati; infatti Adams ha esaminato i tassi di TSH e fT4 durante la prima settimana di vita, Clark dopo la prima settimana di vita e Williams nel cordone e dopo la prima settimana di vita.

Noi abbiamo analizzato esclusivamente soggetti AGA e una popolazione molto più ampia a tutte le età gestazionali considerate ed in particolare alle più basse EG, rispetto alle popolazioni analizzate dagli altri $\mathrm{AA}$. Abbiamo preferito prendere in considerazione i risultati dello Screening effettuato per l'Ipotiroidismo Congenito in $2^{\circ}-3^{\circ}$ giornata di vita per due differenti motivi. Il primo motivo è legato al fatto che, è chiaramente dimostrato, che molti fattori possono interferire sulla secrezione di T4 e soprattutto di TSH al momento della nascita e quindi alterare i livelli di tali ormoni nel sangue del cordone ombelicale $[21,22,28]$. Inoltre i vari fenomeni di adattamento neonatale si accompagnano ad uno spiccato incremento del TSH e del T4 nelle prime 24 ore di vita, con incrementi maggiori in caso di vari distress, soprattutto nel neonato ELGAN, il cui adattamento risulta più difficoltoso rispetto a quello del neonato a termine. In $2^{\circ}-3^{\circ}$ giornata di vita gli effetti dell'adattamento neonatale tendono ad essersi stabilizzati, così che i livelli ormonali risultano simili ai valori 
rilevati nei giorni successivi. Il secondo motivo è giustificato dal fatto che alcuni AA. propongono di effettuare la profilassi per l'Ipotiroidismo Transitorio del neonato ELGAN a partire dal $2^{\circ}-3^{\circ}$ giorno di vita [12-20].

I nostri dati indicano che in $2^{\circ}-3^{\circ}$ giornata il neonato pretermine AGA presenta livelli di tT4 significativamente inferiori rispetto al neonato a termine (Tabella 1). I livelli di tT4 aumentano progressivamente con l'avanzare dell'EG, raggiungendo i valori più elevati nel neonato a termine di 39-42 wk, come indica la correlazione lineare tra i livelli di tT4 e l'EG, che è risultata altamente significativa $(p=0,001)$. $E^{\prime}$ importante sottolineare che l'incremento del tT4 è risultato altamente significativo anche per fasce di età gestazionale di due settimane. Quindi anche un minimo incremento di EG, si accompagna ad un significativo incremento di $\mathrm{TH}$ in tutte le fasce di età e persino nei neonati ELGAN. L'unica eccezione a questo fenomeno è presente nella fascia di età 35-36 settimane, in cui i livelli di tT4 rimangono stazionari rispetto alla fascia d'età precedente; questo riscontro si potrebbe spiegare considerando che questi neonati sono risultati più medicalizzati (antibiotici, nutrizione parenterale totale, trasfusioni) rispetto a quelli di 33-34 settimane. Si potrebbe così ipotizzare che sia la maggior morbidità di base che l'utilizzo di farmaci possano aver interferito con la produzione e/o il rilascio di ormoni tiroidei.

Il TSH mostra invece una più debole correlazione con l'EG, sia nei neonati pretermine che a termine: vi è infatti una tendenza all'incremento dei valori del TSH all'avanzare dell'EG, ma tali aumenti risultano significativi solo in due fasce di età gestazionale $(33-34 \mathrm{wk} v \mathrm{~s}$ $35-36 w k / 37-38 w k$ vs 39-42wk). Noi riteniamo che per poter valutare la funzionalità tiroidea di bambini sottoposti a fattori stressanti, quali sono quelli da noi esaminati, assume più importanza la valutazione del tT4 rispetto al TSH; dal momento che è stato provato che la semplice puntura del tallone innalza i livelli di TSH $[21,22,28,35,36]$.

In relazione ai dati da noi ottenuti, che dimostrano un incremento significativo dei livelli di tT4 anche per piccoli aumenti dell'EG, non riteniamo opportuno iniziare il trattamento ormonale sostitutivo del neonato ELGAN in $2^{\circ}-3^{\circ}$ giornata di vita. Tuttavia è determinante per il clinico avere un punto di riferimento preciso da cui partire, per stabilire se i livelli di TSH e T4 di un determinato bambino incrementano o diminuiscono nei giorni successivi alla nascita. I lavori svolti, concernenti la somministrazione di ormoni tiroidei alla nascita nel neonato pretermine, hanno dimostrato risultati discordanti, per quanto riguarda sia il deficit neurocognitivo di tali soggetti, sia l'incidenza di distress respiratorio e di BPD [11-20]. Attualmente infatti non esistono indicazioni precise, in rapporto agli studi effettuati, per somministrare gli ormoni tiroidei a tutti i soggetti ELGAN. Secondo studi di Williams e coll. soltanto il $14 \%$ dei neonati pretermine va incontro ad un Ipotiroidismo Transitorio durante il primo mese di vita. Poiché vari fattori, quali sepsi, insufficienza renale, insufficienza cardiaca e vari farmaci, possono alterare la funzionalità sia ipofisaria che tiroidea, noi riteniamo che sia utile rivalutare i livelli di TSH e T4 dopo circa 7 giorni dalla nascita ed effettuare la somministrazione di Tiroxina qualora tali livelli risultino inferiori alle 2 DS rispetto ai valori medi da noi ottenuti in $2^{\circ}-3^{\circ}$ giornata di vita. $E^{\prime}$ infatti evidente che si può parlare di carenza ormonale quando i livelli di TSH e T4 siano non inferiori rispetto a quelli del neonato a termine, ma significativamente inferiori a quelli riscontrati in una popolazione sufficientemente ampia di soggetti pretermine di pari EG. La mancata somministrazione di ormoni tiroidei, in caso di necessità, comporta dei rischi che determinano una diminuzione della produzione di surfactante da parte del neonato pretermine, un' alterata termogenesi ed un alterato metabolismo in senso lato, che possono determinare una diminuzione della sopravvivenza del neonato prematuro. Nelle epoche successive, in relazione all'importanza degli ormoni tiroidei sullo sviluppo cerebrale, la mancata somministrazione di questi in un soggetto carente, per un periodo di tempo prolungato, quasi certamente può indurre dei danni al SNC, che si esprimono con un deficit dei processi neurocognitivi del soggetto e con un ridotto QI [37-40].

In conclusione secondo il nostro parere, il feto ed il neonato, anche se pretermine, producono quantità di ormoni sufficienti per sopperire alle loro necessità fisiologiche. Bisogna in ogni caso considerare che, vari fattori che intervengono prima o immediatamente dopo la nascita, possono determinare un esaurimento dell' asse ipotalamo-ipofisi-tiroide ed è in tale momento che dovremo sopperire alle necessità del piccolo essere, somministrando ciò che la natura non è più in grado di produrre a sufficienza.

\section{BIBLIOGRAFIA}

1. Fisher DA. Thyroid function and dysfunction in premature infants. Pediatr Endocrinol Rev 2007;4:317-28.

2. Fisher DA. Thyroid system immaturities in very low birth weight. Semin Perinatol 2008;32:387-97.

3. La Franchi $S$. Thyroid function in the preterm infant. Thyroid 1999;9:71-8.

4. Paul DA, Leef KH, Stefano JL, Bartoshesky L. Low serum thyroxine on initial newborn screening is associated with intraventricular hemorrahage and death in very low birth weight infants. Pediatrics 1998;101:903-7.

5. Simic N, Asztalos EV, Rovet J. Impact of neonatal thyroid hormone insufficiency and medical morbidity on infant neurodevelopment and attention following preterm birth. Thyroid 2009;19:395-401.

6. Paul DA, Mackley A, Yencha EM. Thyroid function in term and late preterm infants with respiratory distress in relation to severity of illness. Thyroid 2010;20:189-94.

7. Kuint J, Sack J, Maayan-Metzger A. Early blood thyroxine concentration and necrotizing enterocolitis in premature infants. Acta Paediatr 2008;97:304-7. 
8. Reuss ML, Paneth N, Lorenz JM, Susser M. Correlates of low thyroxine values at newborn screening among infants born before 32 weeks gestation. Early Hum Dev 1997;47:223-33.

9. Reuss ML, Paneth N, Pinto-Martin JA, et al. The relation of transient hypothyroxinemia in preterm infants to neurologic development at two years of age. New Engl J Med 1996;334:821-7.

10. Fisher DA. Hypothyroxinemia in premature infants: is thyroxine treatment necessary? Thyroid 1999;9:715-20.

11. La Gamma EF, Paneth N. Clinical importance of hypothyroxinemia in the preterm infant and discussion of treatment concerns. Curr Opin Pediatr 2012;24:172-80.

12. La Gamma EF, van Wassenaer AG, Ares $S$, et al. Phase 1 trial of 4 thyroid hormone regimens for transient hypothyroxinemia in neonates of <28 weeks' gestation. Pediatrics 2009; 124:e258-68.

13. Wassenaer AG, Westera J, Houtzager BA, Kok JH. Ten-year follow-up of children born at $<30$ weeks' gestational age supplemented with thyroxine in the neonatal period in a randomized, controlled trial. Pediatrics 2005;116:e613-8.

14. Van Wassenaer AG, Kok JH. Trial with thyroid hormone in preterm infants: clinical and neurodevelopment effects. Semin Perinatol 2008:32:423-30.

15. Vanhole C, Aerssens $P$, Naulaers $G$, et al. L-thyroxine treatment of preterm newborns: clinical and endocrine effects. Pediatr Res 1997; 42:87-92.

16. Van Wassenaer AG, Kok JH, Dekker FW, et al. Thyroxine administration to infants of less than 30 weeks age decreases plasma triiodothyronine concentrations. Eur J Endocrinol 1998;139:508-15.

17. Osborn DA, Hunt RW. Prophylactic postnatal thyroid hormones for prevention of morbidity and mortality in preterm infants. Cochrane Database Syst Rev 2007:CD005948.

18. Osborn DA, Hunt RW. Postnatal thyroid hormones for respiratory distress syndrome in preterm infants. Cochrane Database Syst Rev 2007:CD005946.

19. Van Wassenaer AG, Kok JH, de Vijlder JJ, et al. Effects of thyroxine supplementation on neurologic development in infants born at less than 30 weeks' gestation. N Engl J Med 1997;336:21-6.

20. Amato M, Pasquier S, Carasso A, Von Muralt G. Postnatal thyroxine administration for idiopathic respiratory distress syndrome in preterm infants. Horm Res 1988;29:27-30.

21. Gemer O, Shenhav S, Segal S, Tur-Kaspa I. Thyroid hormone levels in cord blood of infants with academia at birth. Eur J Obstet Gynecol Reprod Biol 2000;93:53-5.

22. Williams FL, Hume R. Perinatal factors affecting thyroid hormone status in extreme preterm infants. Semin Perinatol 2008;32:398-402.

23. Ballabio M, Nicolini $U$, Jowett T, et al. Maturation of thyroid function in normal human foetuses. Clin Endocrinol (Oxf) 1989;31:565-71.

24. Thorpe-Beeston JG, Nicolaides KH, Felton CV, et al. Maturation of the secretion of thyroid hormone and thyroid-stimulating hormone in the fetus. N Engl J Med 1991;324:532-6.

25. Kilby MD, Verhaeg J, Gittoes M, et al. Circulating thyroid hormone concentrations and placental thyroid hormone receptor expression in normal human pregnancy and pregnancy complicated by intrauterine growth restriction (IUGR.) J Clin Endocrinol Metab 1998:83:2964-71.

26. Klein AH, Oddie TH, Parslow M, et al. Developmental changes in pituitary-thyroid function in the human fetus and newborn. Early Hum Dev 1982;6:321-30.

27. Bongers-Schokking JJ, Schopman W. Thyroid function in healthy normal, low birthweight and preterm infants. Eur J Pediatr 1984;143:117-22.

28. Rashmi, Seth A, Sekhri T, Agarwal A. Effect of perinatal factors on cord blood thyroid stimulating hormone levels. J Pediatr Endocrinol Metab 2007;20:59-64.

29. Van Wassenaer AG, Kok JH, Dekker FW, De Vijder JJ. Thyroid function in very preterm infants: influences of gestational age and disease. Pediatr Res 1997;42:604-9.

30. Biswas S, Buffery J, Enoch H, et al. A longitudinal assessment of thyroid hormone concentrations in preterm infants younger than 30 weeks' gestation during the first 2 weeks of life and their relationship to outcome. Pediatrics 2002;109:222-7.

31. Dilli D, Ouz SS, Andiran N, et al. Serum thyroid levels in preterm infants born before 33 weeks of gestation and association of transient hypothyroxinemia with postnatal characteristics. J. Pediatr Endocrinol Metabol 2010;23:899-912.

32. Adams, MD, Emery, MD, Clark, MD, et al. Reference ranges for newer thyroid function tests in premature infants. J Pediatr 1995;126:122-7.

33. Clark SJ, Deming DD, Emery JR, et al. Reference ranges for thyroid function tests in premature infants beyond the first week of life. $J$ Perinatol 2001:21:531-6.

34. Williams FL, Simpson J, Delahunty C, et al. Developmental trends in cord and postpartum serum thyroid hormones in preterm infants. J Clin Endocrinol Metab 2004;89:5314-20.

35. Williams FL, Mires GJ, Barnett C, et al. Transient hypothyroxinemia in preterm infants: the role of cord sera thyroid hormone levels adjusted for prenatal and intrapartum factors. J Clin Endocrinol Metab 2005;90:4599-606.

36. Ng SM, Wong SC, Paize F, et al. Multivariate analyses of factors that affect neonatal screening thyroid stimulating hormone. $J$ Pediatr Endocrinol Metab 2011;24:727-32.

37. Huo $K$, Zhang Z, Zhao D, et al. Risk factors for neurodevelopmental deficits in congenital hypothyroidism after early substitution treatment. Endocr J 2011;58:355-61.

38. Huang CB, Chen FS, Chung MY. Transient hypothyroxinemia of prematurity is associated with abnormal cranial ultrasound and illness severity. Am J Perinatol 2002;19:139-47.

39. Williams GR. Neurodevelopmental and neurophysiological actions of thyroid hormone. J Neuroendocrinol 2008;20:784-94.

40. Bernal J. Thyroid hormones and brain development. Vitam Horm 2005:71:95-122. 